\title{
Investigation on the Interaction of Secondary Current Pulses with the Main Wavefront
}

\author{
Rosy Balaram Raysaha* and Udaya Kumar
}

High Voltage Laboratory, Department of Electrical Engineering, Indian Institute of Science, Bangalore, India

\begin{abstract}
Additional current pulses get injected into the lightning channel from branches and/or from successive reflections within struck Tall Grounded Objects (TGOs). These pulses termed here as "secondary current waves/pulses" affect the lightning Electro-Magnetic Fields (EMFs). With regard to these, an important question arises on whether or not these pulses get reflected at the main wavefront and there seems to be uncertainty in the pertinent literature. A particular case involving only the first ground end reflection from a struck TGO has been fully dealt in our earlier work. However, the same for subsequent reflections from TGO and that for the current fed by the branches were not dealt and this forms the main goal of present work. As the main objective is to ascertain the status of these secondary current pulses after they have reached the main wavefront, dynamics at the point of injection assumes lesser importance. In view of this, a lumped voltage source is employed for the injection of secondary current pulses at appropriate time instants. The main stroke evolution is emulated by a macroscopic physical model, which was developed in our earlier work. Investigation showed that these secondary pulses merge with the main wavefront without any sign of reflection whatsoever. Analysis showed that the spatial dynamic resistance/conductance profile at the main wavefront is basically responsible for the same with distributed source providing additional support. These findings are in line with the observations made in our earlier work for the first ground end reflected current.
\end{abstract}

Keywords: Corona sheath, lightning return stroke, non-linear spatio-temporal resistance profile, reflection at main wavefront.

\section{INTRODUCTION}

Lightning hazards to electrical systems can be traced to the stroke current and the associated Electro-Magnetic Fields (EMFs). The latter is governed by the spatio-temporal distribution of stroke current along the channel. The main stroke current is modulated by additional current pulses arising from: (a) feeding to the branches and (b) reflected current pulses transmitted onto the channel during strike to Tall Ground based Objects (TGOs). These additional pulses are termed as "secondary current pulses" in this work. Well before the injection of these secondary pulses at any point, the main wavefront would have traversed ahead converting the nascent channel into a highly conducting channel. In other words, till these pulses catch up with the main wavefront, they will be propagating through a channel having high conductance. Due to this, it is envisaged in the literature that these secondary current pulses propagate with a full velocity $c$ i.e., velocity of light in free space. As a consequence, several questions arise: (i) would these pulses get reflected at the main wavefront, (ii) if so, how long will they survive on the channel and (iii) is there any polarity dependency.

Many works have dealt with lightning strike to TGOs and a review of the same has been presented in $[1,2]$. The analysis was based on engineering models [3-8], distributed circuit models [9] and electromagnetic models [10-14].

*Address correspondence to these authors at the High Voltage Laboratory, Department of Electrical Engineering, Indian Institute of Science, Bangalore, India; Tel: +91-080-22933177; Fax: +91-080-22932373;

E-mails: rosy@ee.iisc.ernet.in, uday@hve.iisc.ernet.in
In these works, the dynamics at the main wavefront for the secondary current pulses arising out of successive reflection within a TGO was not the issue under consideration.

Further, the use of lumped source in some distributed circuit and electromagnetic models, even after incorporation of non-linearity, does not adequately facilitate evaluation of the dynamics at the main wavefront. On the other hand, in engineering models, either the secondary current pulses are assumed to disappear at the main wavefront introducing a discontinuity in current [8] or it is assumed to get reflected at the main wavefront. However, no adequate justification is provided for either case. In view of these, the present work aims to investigate on the dynamics at the main wavefront for the secondary current pulses arising out of TGO and branching.

Deriving answers to the questions (i-iii) through field experimentation would be quite involved and further, it is only the EMFs rather than the actual current in the channel that can be measured. In view of these, theoretical modeling approaches, which can emulate return stroke evolution, seem to be the ideal choice. Amongst different kinds of lightning return stroke models [15-20], only distributed circuit models $[9,21-24]$ and electromagnetic models [10-14, 25-28] have an ability to trace return stroke current evolution. However, distributed circuit models suffer from an inherent limitation arising due to the quasi-TEM mode approximation for the current wave propagation. In addition, there are inconsistencies in the approaches employed for deducing the associated parameters and its extension to model the strike to TGOs. On the other hand, electromagnetic models simulate return stroke current evolution by solving the governing field equations without resorting to any simplification of the field 
structure i.e., no assumption is made on the mode of propagation. This feature makes it best suited to model the propagation of return stroke current, as well as, to consider strike to TGO.

In our considered opinion, any attempt towards a more realistic physical modeling of the return stroke current evolution requires appropriate accounting of: (a) the excitation formed by electric field due to the initial charge distribution along the channel, that of cloud and that induced on the ground, (b) the transient enhancement of conductance by several orders at the bridging regime, which initiates the return stroke, (c) the non-linear variation in channel conductance and (d) the associated dynamic EMFs that support the current evolution. A few of the distributed circuit models, for example [21, 22], have attempted to consider some or many of these aspects. However, they suffer from the inherent limitations listed above. On the other hand, most of the electromagnetic models employ lumped sources for the required excitation and hence, the need for modeling basic channel dynamics did not arise. In the absence of such a modeling capability, they cannot be relied for the intended task. Incidentally, a macroscopic physical model for the lightning return stroke incorporating the features (a-d), has been successfully developed in our earlier work [29]. The same will be employed here for depicting the main stroke evolution.

In our earlier work [30, 31], analysis for the first ground end reflected wave from struck TGO was dealt without resorting to any artificial current injection. It was shown that this secondary current pulse merges with the main wavefront without any sign of reflection. However, secondary current pulses arising due to subsequent reflections within TGO and that fed by branching was not addressed and this forms the main goal of present work.

\section{MODELING DETAILS}

As mentioned in section I, a macroscopic physical model developed in our earlier work [29] will be employed for the modeling of main stroke evolution. The complete details of the same along with model predicted results can be found in [29]. However, for sake of completeness, a brief description of the same will be provided below:

\section{A. Macroscopic Physical Return Stroke Model}

The time-domain electromagnetic model [29], which is termed as "Macroscopic Physical Model", incorporates with suitable approximations all the essential physical processes (a-d) governing the return stroke evolution. The characteristics of model predicted spatio-temporal current distribution, as well as, the resulting EMFs from $50 \mathrm{~m}$ to 200 $\mathrm{km}$ exhibited excellent matching with the field data. In view of this, it can be entrusted that it has the ability to provide answers to the questions raised in section I. However, at present, the model deals only with strike to ground scenario [29].

The required field computation in this modeling comprises of evaluation of initial charge distribution and dynamic EMFs associated with the return stroke. For the calculation of pre-return stroke charge distribution along the channel, Charge Simulation Method (CSM) is employed [29]. The participating charge centre of the cloud is approximated to be spherical and the straight, vertical channel is assumed to begin from its bottom. The radius and magnitude of this spherical charge is calculated by specifying channel length, channel root potential and gradient at its surface. The corona sheath surrounding the channel core is assumed to have formed during the leader phase and its radius is evaluated iteratively [29]. However, it is made time invariant in the return stroke model so as to minimize computational burden. For the numerical solution of dynamic EMFs, a Time Domain Electric Field Integral Equation (TD-EFIE) [32] is adopted.

For the evaluation of dynamic conductance at the bridging/streamer regime, Toepler's spark law is employed [29]. A first order arc model is adopted to describe the nonlinear channel conductance at the matured channel section [29]. The arc time constant for rising current, in an attempt to make them current dependent, is made to vary with altitude. Also, the settling value of conductance is made a function of instantaneous current. Further, the steady-state arc gradient, as well as, the critical gradient for corona inception is subjected to air density and temperature correction factor. The conductivity of corona sheath is made field dependent.

\section{B. Secondary Current Pulse Injection}

The secondary current pulses, as discussed in section I, originates from branching and successive reflection within TGO. Incorporation of this in the macroscopic physical model requires: (i) a time domain code for the numerical solution of EFIE in 3D (required for handling branches) and (ii) a suitable formulation to accurately handle the abrupt change in cross-section (or in simplified version, the radius) in thin wire TD-EFIE. The former was not dealt in our earlier work [29] while, the required formulation in time domain for the latter is rather difficult to find. At the same time, the dynamics at the point of injection can be expected to have limited influence at the distant wavefront region. Due to these, a simplified approach is adopted for the realization of secondary current pulses. Accordingly, a suitable lumped voltage source is inserted in series with the channel. In other words, the inception and evolution of main stroke current perfectly follows the associated physical processes and it is only the injection process of secondary current pulse, which deviates from reality.

The main objections for the use of a lumped source for secondary current pulse injection are: (i) it injects equal currents in both directions and (ii) the charge-current relation (at least) across the source is dictated by the source strength and it is dipolar in nature. Both these scenarios are different from the reality. However, as mentioned earlier, the dynamics at the point of injection is expected to have minor influence at the distant wavefront and hence, these limitations can be tolerated. It is worth to recall here that our earlier work [30, 31], which is limited to a special case of $\mathrm{TGO}$, is free of any such assumptions/limitations.

The current injected into the channel by successively reflected current within TGO can be expected to have a waveshape very similar to the main stroke current. This approximation neglects the TM mode of current propagation on TGO and hence, the corresponding modification of reflection coefficients with the frequency contents of the 
current $[31,33]$. Nevertheless, it is still adequate for the intended task of ascertaining the dynamics at the main wavefront for the secondary current pulses. Even though the wave shape of the actual current injected by the branch is expected to be quite different from the main pulse, it is set to be the same for simplicity. In other words, the required waveshape for the secondary current pulse is taken from the base current of corresponding strike to ground scenario (refer Fig. 1) and its magnitude is altered as per the requirement.

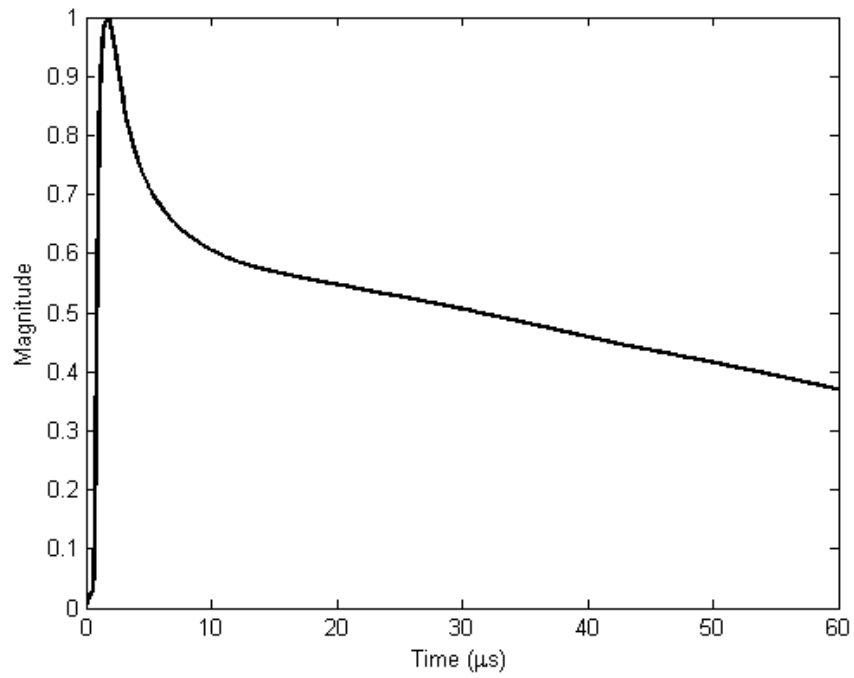

Fig. (1). Normalized voltage waveform injected by the lumped voltage source.

\section{SIMULATIONS FOR DYNAMICS AT MAIN WAVEFRONT}

The macroscopic physical model, which is employed for the emulation of main stroke evolution, has fully incorporated all the essential physical process governing the phenomena. In view of this, it is expected that the dynamics for the secondary current pulses at the main wavefront is reliably depicted. The basic parameters used in this macroscopic physical model for a downward cloud-toground strike will be presented below.

Observing that negative lightning is more common, simulations are basically intended for a downward cloud-toground negative flash. Accordingly, the streamer gradient is set to $400 \mathrm{kV} / \mathrm{m} \mathrm{[34]} \mathrm{and} \mathrm{the} \mathrm{leader} \mathrm{gradient} \mathrm{is} \mathrm{set} \mathrm{to} 6 \mathrm{kV} / \mathrm{m}$ [35]. The spatial discretization length is $20 \mathrm{~m}$ and the channel core radius is set to $2.5 \mathrm{~mm}[15,16,36]$. The values of other parameters are same as mentioned in [29]. As the distinction between the conditions prevailing before the positive and negative return stroke lies in their respective streamer gradients, the results presented is believed to be independent of the stroke polarity.

The range of channel length and cloud base potential considered are $4.5-9 \mathrm{~km}$ and $50-150 \mathrm{MV}$ respectively. However, for brevity sample simulation results corresponding to a channel length of $6 \mathrm{~km}$ and cloud base potential of $52 \mathrm{MV}$, which yields a current of $30 \mathrm{kA}$, will only be presented. As mentioned in the previous section, for the injection of secondary current pulse, a series lumped voltage source is employed with its temporal variation set to follow that of the base current during strike to ground scenario. A range of source voltage levels, which injects peak current amplitudes in the requisite range are considered.

\section{A. Secondary Current Pulse Due to Branching}

For emulating the scenario of secondary current pulse injection due to a branch, the lumped voltage source described above, is placed at the point of branching. The corresponding time instant of pulse injection $t_{i n j}$ is calculated as: $t_{i n j}=t_{r}+t_{b}$ where, $t_{b}$ is one way travel time on the branch and $t_{r}$ is the time taken to reach the branching point from the attachment point, which was estimated from strike to ground case. In fact, this travel time is considered only to avoid injection at the wavefront itself, which will defeat the very purpose of examining any reflection. Current pulses of different magnitudes and points of injection were considered.

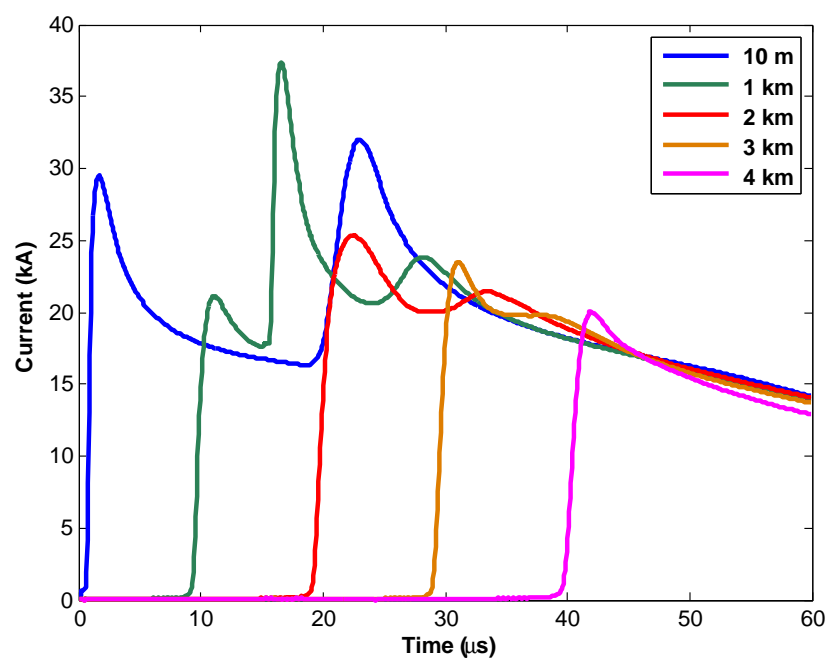

Fig. (2). Simulation results for current injected by a branch of length $600 \mathrm{~m}$ with branching point located at $1 \mathrm{~km}$ above ground.

Sample simulation result, which corresponds to a branch length of $600 \mathrm{~m}$ located at $1 \mathrm{~km}$ above the ground, is presented in Fig. (2). By assuming a propagation velocity of $c / 3$ along the branch, the time of injection amounts to $15 \mu \mathrm{s}$. The strength of the lumped voltage source was $0.7 \mathrm{MV}$, which injected a current of about $20 \mathrm{kA}$. It can be seen from the figure that the injected current pulse propagates in both the directions with attenuation and dispersion before finally merging with the main wavefront. In other words, there exists no reflection of the secondary current pulse at the main wavefront. These observations remained true for a wide range of branching positions, branch lengths and injected currents.

\section{B. Secondary Current Pulse Due to Successive Reflection within TGO}

Before entering into the details of successive reflection, it is worth recalling here that the attachment point during strike to a TGO is quite elevated from the TGO top. As a result, the current initiated at the junction of upward and downward discharges propagate in both the directions [30]. The downward moving current wave encounters a reflection at the TGO-channel junction, however, it is not considered in the present analysis. It will be evident later in this section that the findings of the work are not limited by the method employed for the evaluation of attachment point, as it is not 
dependent on the time of arrival of reflected waves. In view of this, for simplicity in formulation, Eriksson's formula [37] is employed for the estimation of height of attachment point.

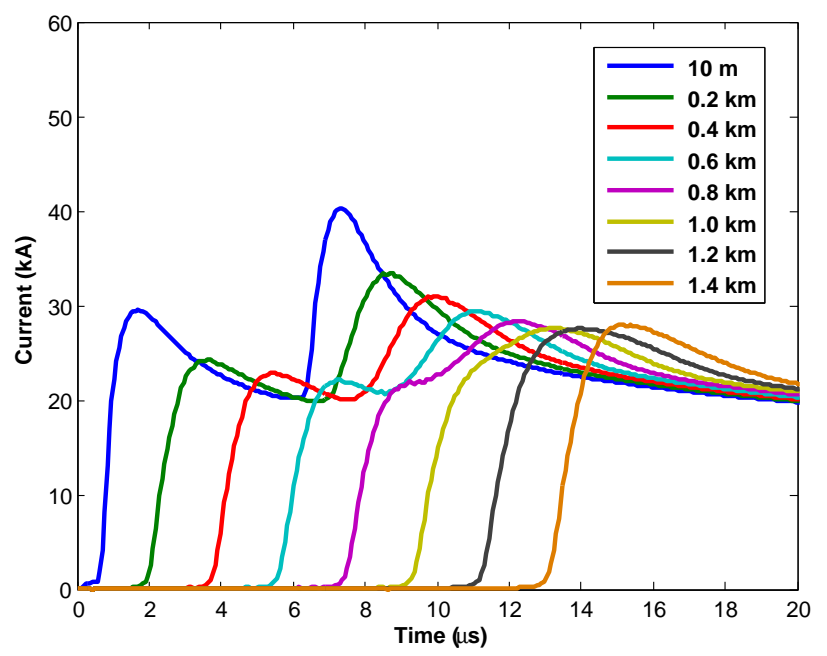

Fig. (3). Simulation results for first ground end reflection during strike to a $560 \mathrm{~m}$ TGO.

Two distinct scenarios are to be considered with reflections originating from ground end of TGO. The first scenario corresponds to the positive reflected waves, which accounts for odd number (first, third, fifth, etc.,) of reflections from ground while, the second one corresponds to the negative reflected wave arising from even number of reflections. This polarity reversal is due to the negative reflection from TGO top (while, that at ground end is positive). As the goal is to verify whether reflection of secondary current wave occurs at the main wavefront, no efforts are made to correct the magnitude of subsequent reflections in the current injection. The time of secondary current pulse injection is estimated as: $t_{i n j}=t_{t}+(2 n-1) * h / c$ where, $n$ is the order of reflection, $t_{t}$ is the time taken to reach the TGO top from the attachment point (calculated with velocity of $c / 3$ ). The attachment point for TGOs of height $168 \mathrm{~m}$ and $560 \mathrm{~m}$, for which sample results will be presented in this section, are located at $180 \mathrm{~m}$ and $370 \mathrm{~m}$ above TGO respectively. These values apply for the selected channel root potential of $52 \mathrm{MV}$.

As mentioned in section I, analysis for a secondary current pulse originating from the first ground end reflection of a TGO has been considered in [30]. It is worth recalling here that no simplification was adopted for the secondary current injection in [30] and hence, it was free of any associated limitations. For the sake of comparison, first ground end reflection from a $560 \mathrm{~m}$ TGO is analyzed here. The corresponding simulation result is presented in Fig. (3) wherein the time of injection is $5.7 \mu \mathrm{s}$ and the source strength employed is $0.4 \mathrm{MV}$. It is evident that in line with the findings of the earlier work, the secondary current pulse does not get reflected at the main wavefront.

Next the analysis for the secondary current pulses injected by subsequent reflection is considered. To check the generality of the results, analysis is carried out for TGOs of different heights and for brevity, only results corresponding to $168 \mathrm{~m}$ TGO will be presented. The results for second and third ground end reflections are presented in Fig. $(\mathbf{4 a}, \mathbf{b})$ respectively. The second ground end reflection is of opposite polarity due to the negative reflection from TGO top, which forms the incident wave. Owing to the same reason, the third ground end reflection is of positive polarity. Obviously, the current injected into the channel by every successively reflected wave will be of the same polarity as the ground end reflected wave. The source strength employed for both the cases was $0.3 \mathrm{MV}$ and the corresponding time of injection are $3.5 \mu$ s and $4.6 \mu$ s respectively. It is clearly evident form the figure that there exists no reflection of the secondary current pulses at the main wavefront. This observation remained true for a wide range of TGO heights and current levels.

(a) Second ground end reflection

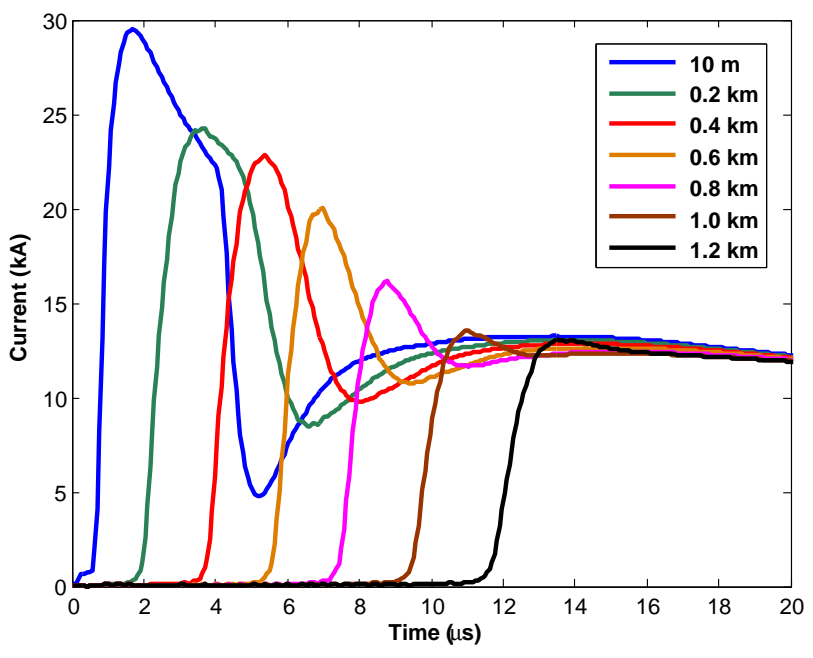

(b) Third ground end reflection

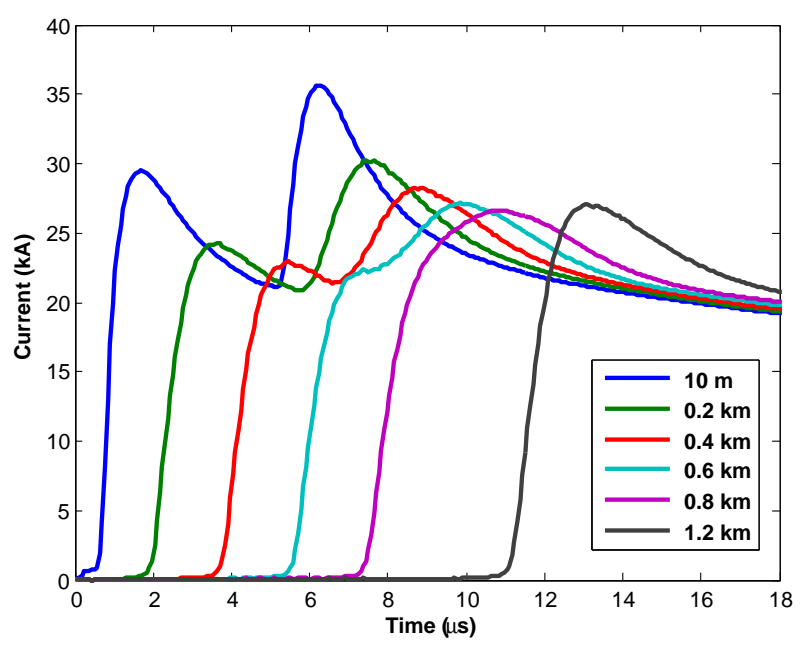

Fig. (4). Simulation results for second and third ground end reflection during strike to a $168 \mathrm{~m}$ TGO.

As stated above, in our earlier work [30] no artificial current injection was employed. It is important to note that whole of the physical processes were fully represented. On the other hand, for the reasons quoted earlier, the present work has employed an artificial mechanism for current injection. Therefore, it would be interesting to compare the two cases so as to ascertain the differences in secondary 
current propagation. From a comparison, it is found that, the rate of attenuation of current amplitude seems to be relatively higher in the present case. Except for this, no other serious differences could be seen. As the analysis in the present case is carried out for a range of current pulses (of magnitudes even comparable to the main current), the above has not limited the validity of the main results. Therefore, the conclusion drawn is not limited by the simplification adopted for secondary current injection.

\section{ANALYSIS FOR MERGING OF SECONDARY CURRENT PULSES AT MAIN WAVEFRONT}

As shown in previous section, the upward propagating secondary current wave on the channel merges with the main wavefront without any sign of reflection whatsoever. The possible sources, which can contribute to this phenomenon, are: (i) corona sheath, (ii) non-linear variation of channel conductance/resistance at the main wavefront and (iii) distributed source. In our earlier work [30], these have been investigated in detail, however, only for a special case involving equal TGO and channel core radii. As the time of arrival of the secondary current pulses, their strength and polarity can be different in the present case, whole of the investigation is independently carried out and the same will be discussed below.

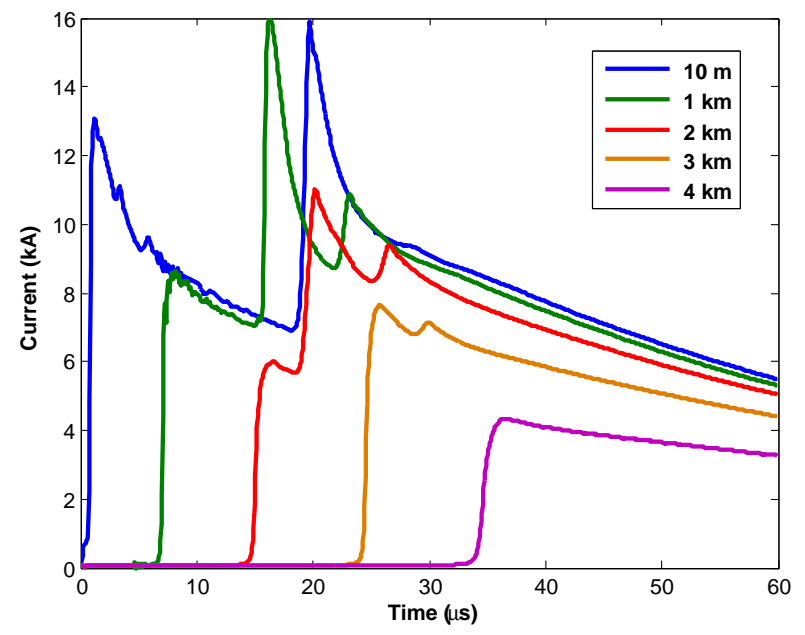

Fig. (5). Temporal variation of current excluding corona sheath for the case presented in Fig. (2).

To construe the effect of corona sheath, simulations are run excluding it and the results obtained are presented in Figs. $(\mathbf{5}, \mathbf{6})$ for the cases dealt in Figs. $(\mathbf{2}, \mathbf{3})$ respectively. It is evident from the figures that the upward moving secondary current wave does not get reflected at the main wavefront even in the absence of corona sheath. However, it can be verified that absence of corona sheath leads to an increase in velocity and decrease in magnitude of the main stroke current. Based on the above, it is concluded that corona sheath is not responsible for the merging of secondary current pulses at the main wavefront.

Next the role of channel conductance/resistance is analyzed. As the goal here is to isolate the possible role of channel resistance, other aspects of return stroke evolution like role of corona sheath, distributed excitation/source etc., are not to be included. In view of this, a suitably loaded monopole with lumped excitation at its ground end would be an ideal choice for the intended investigation. Accordingly, lower half of the monopole is loaded with $0.2 \Omega / \mathrm{m}$ (typical resistance per unit length of matured channel) and the upper half with $60 \Omega / \mathrm{m}$ (typical resistance per unit length of nascent channel) $[15,16]$.

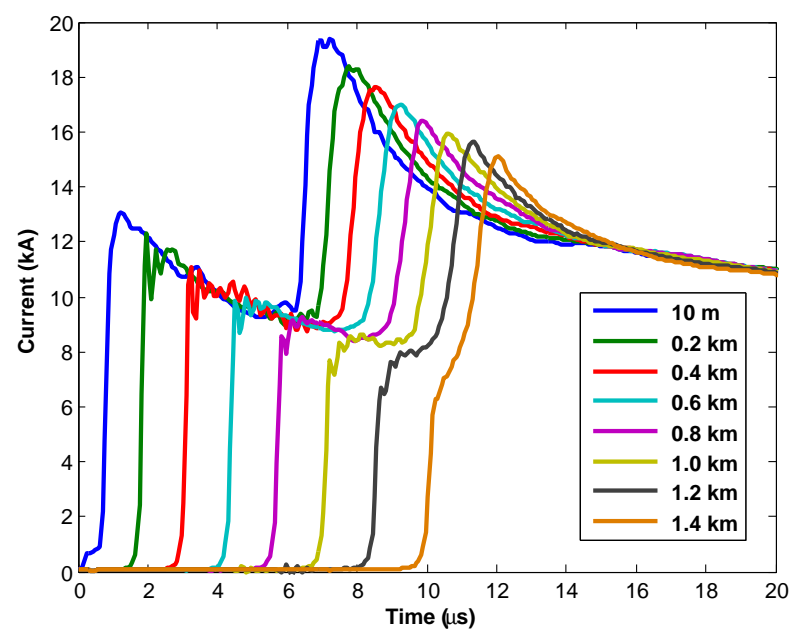

Fig. (6). Temporal variation of current excluding corona sheath for the case presented in Fig. (3).

The spatial resistance profile at the junction is to be set such that it emulates the resistance distribution at the main wavefront region. For this, three profiles are considered: (a) step profile, (b) Gaussian half-wave profile and (c) moving Gaussian half-wave profile. The first profile corresponds to treating wavefront as a sharp discontinuity while, the remaining two envisages a smooth transition from a high conducting state to a low conducting state. The last two profiles are derived from the simulation results of our earlier work [29]. It was observed that the spatial resistance profile at the wavefront closely resembled the rising portion of the Gaussian function of the form $\exp \left(-b\left(z-z_{o}\right)^{2}\right)$, which in this work has been termed as "Gaussian half-wave profile". In other words, the resistance increases from $0.2 \Omega / \mathrm{m}$ to 60 $\Omega / \mathrm{m}$ within a spatial span of about $200 \mathrm{~m}$. Noting that the wavefront and hence, the resistance profile at the junction propagates along the channel, case (c) is considered. Incidentally, results presented in [30] indicated that 0.5 is the approximate velocity ratio of main wavefront to secondary wavefront. In view of this, for (c) the spatial resistance profile is made to move with a velocity of $c / 2$ (once the current reaches the junction) in the upward direction.

For a clear identification of reflection, a waveshape with a suitable front and limited time span [30] is essential. Otherwise, the arrival of reflection will modify the tail portion of the current and hence, complicate the quantification. Considering these, a suitable Gaussian pulse was employed in the previous work [30]. The same was employed in the present work for an initial estimation. However, it is observed that for TM mode, the reflection can be a function of frequency components of the incident current $[31,33]$ and therefore, use of actual current draws some merits. Hence, the analysis is repeated with injected secondary current whose temporal variation is set to follow 
that of the base current during strike to ground scenario (as discussed in section II \& III).

For the analysis, a $2.5 \mathrm{~km}$ long monopole of $2.5 \mathrm{~mm}$ radius is considered. All the simulations are carried out with the numerical code for TDFIE, which is a subset of our return stroke simulation code [29]. For all the cases, the transition of resistance is implemented at the middle of the monopole to enable a clear identification of the reflected current. With regard to the reflection, it is necessary to monitor the current at a point, which: (i) is relatively closer to the junction and (ii) the incident and reflected wave fronts must be clearly separated. Accordingly, a measurement point on the monopole at a height of $0.8 \mathrm{~km}$ above ground i.e., in Fig. (7a-c), third curve from left is chosen. For a clear identification of reflection, the current for uniform loading case $(0.2 \Omega / \mathrm{m}$ throughout $)$ is also provided for the measurement point located at $0.8 \mathrm{~km}$ (red dotted curves in Fig. 7). The time base for the plot is selected such that the reflection from the open end of the monopole is kept away.

From the figures it is evident that the reflection is minimal for the spatial resistance profile (c) while, it is maximum for case (a). These observations are in line with that reported in our earlier work [30] wherein a Gaussian excitation was employed. The quantification of this reflection is rather difficult due to: (i) reflection at the junction is frequency dependent $[31,33]$ and (ii) the current wave propagates with attenuation and dispersion (which can be seen in Fig. 7). Nevertheless, an attempt is made for the estimation of reflection by assuming a loss-less, distortionless current propagation with fixed reflection coefficient and reflecting junction being moving upwards with a velocity of $c / 2$.

For the required estimation, the incident current is obtained by performing the simulation for a uniformly loaded case (with $0.2 \Omega / \mathrm{m}$ loading throughout). This incident current is represented by red dotted curves in Fig. (7). On this current, negative reflection is added with a time delay corresponding to two-way travel time between the selected evaluation point and the junction. This delay starts from $3 \mu \mathrm{s}$ for the first reflection. However, due to the upward propagation of the junction, subsequent reflections get delayed in time. The magnitude of reflection is varied and the resulting waveforms are compared with that obtained for case (c) indicated by red solid curve in Fig. (7c). From this comparison it is found that the magnitude of reflection is within $20 \%$. The reflection with Gaussian excitation can be evaluated directly as the incident and reflected currents are adequately separated. The reflection evaluated with the same is about 15 - $16 \%$. The difference in the magnitude of reflection for either case can be attributed to the frequency content of the incident current and the problem of dispersion, which has noticeable affect on the former.

Based on the above analysis, it is then concluded that the spatial resistance/conductance profile of the channel is the main contributor for collapse or merging of the secondary current waves at the main wavefront. The distributed excitation in the lightning channel, perhaps, contributes in a complex way to eliminate the remnant part of reflection. However, any separate study in this direction is deemed unnecessary as it will be same as the cases considered for the main simulation i.e., section III. (a) Step profile

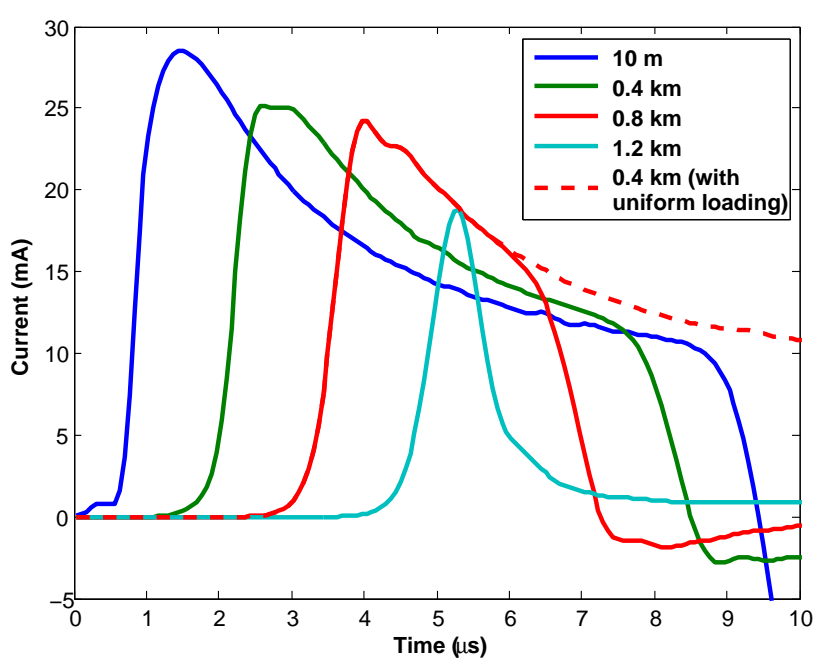

(b) Gaussian half-wave profile

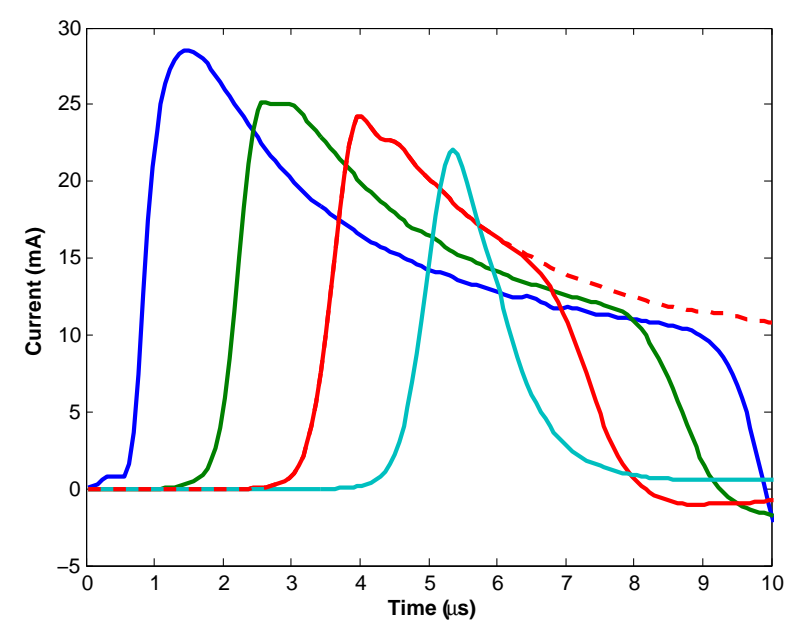

(c) moving Gaussian half-wave profile

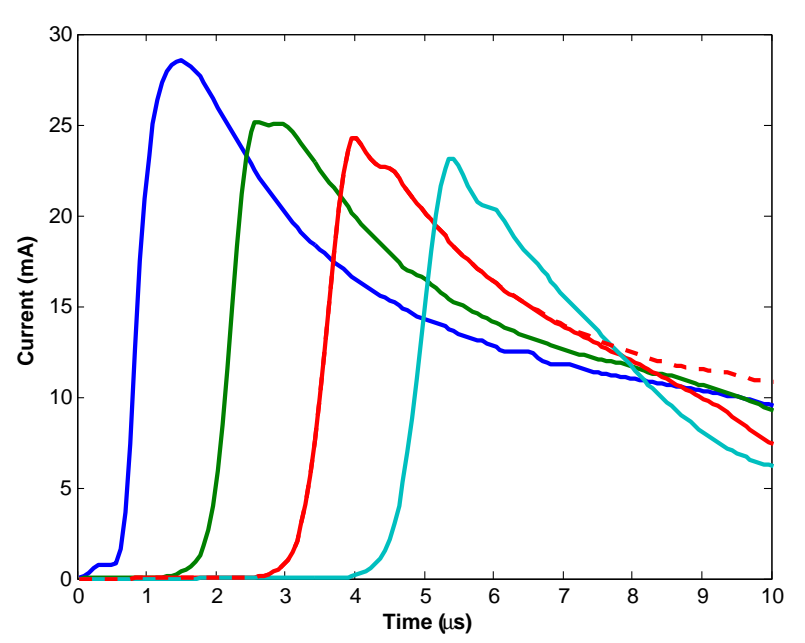

Fig. (7). Temporal current variation for different spatial resistance profile (In all the cases, third curve from left is used for the calculation of $\%$ reflection). 


\section{CONCLUSION}

The lightning main stroke current gets modulated due to the presence of secondary current pulses, which are injected from branches and/or from successive reflections within struck Tall Grounded Objects (TGOs). The knowledge on the final status of these secondary current waves is essential for evaluating lightning EMFs. In this connection, an important issue concerning whether or not the secondary current pulses get reflected at the main wavefront needs to be ascertained. However, limited information on the same could be found in the pertinent literature. The present work has made an attempt to address this vital issue for the secondary current pulses arising from branches and that due to successive reflections within struck TGO.

For the intended analysis, the main stroke current is emulated by employing a macroscopic physical model. Noting that the dynamics at the point of injection has very limited influence on distant wavefront, lumped voltage source is employed for the injection of secondary current pulses. These pulses are deemed to have a waveshape same as that of the incident current. Analysis revealed the following: (i) secondary current waves get smoothly merged at the main wavefront, without any sign of reflection whatsoever, (ii) the dynamic spatial channel resistance profile at the main wavefront is found to be mainly responsible for this phenomenon and (iii) the distributed nature of source is found to give additional support. These results are in line with our earlier work, wherein only a special case of strike to TGO was considered, however, with no artificial means for realization of secondary current.

\section{REFERENCES}

[1] Rakov VA. Transient response of a tall object to lightning. IEEE Trans Electromagn Compatibil 2001; 43: 654-61.

[2] Rachidi F. Modeling lightning return strokes to tall structures. J Light Res 2007; 1: 16-31.

[3] Rachidi F, Rakov VA, Nucci CA, Bermudez JL. Effect of vertically extended strike object on the distribution of current along the lightning channel. J Geophys Res 2002; 107(D23): 4699.

[4] Guerrieri S., Nucci CA, Rachidi F, Rubinstein M. On the influence of elevated strike objects on directly measured and indirectly estimated lightning currents. IEEE Trans Power Deliv 1998; 13(4): 1543-55.

[5] Motoyama H, Janischewskyj W, Hussein AM, Rusan R, Chisholm WA, Chang JS. Electromagnetic field radiation model for lightning strokes to tall structures. IEEE Trans Power Deliv 1996; 11(3): 1624-32.

[6] Rachidi, F, Janischewskyj W, Hussein AM, et al. Current and electromagnetic field associated with lightning return strokes to high towers. IEEE Trans Electromagn Compatibil 2001; 43(3): 356-67.

[7] Bermudez JL, Rubinstein M, Rachidi F, Heidler F, Paolone M. Determination of reflection coefficients at the top and bottom of elevated strike objects struck by lightning. J Geophys Res 2003; 108 (D14), 4413-25.

[8] Pavanello D, Rachidi F, Rubinstein M, Bermudez JL, Nucci CA. Electromagnetic field radiated by lightning to tall towers: Treatment of the discontinuity at the return stroke wavefront. J Geophys Res 2004; 109: D06114, p. 7.

[9] De Conti A, Visacro S. On the use of lumped sources in a nonlinear lightning return stroke model and extension for evaluating strikes to tall objects. J Geophys Res 2009; 114: D11115.

[10] Podgorski AS, Landt JA. Numerical analysis of the lightning-CN tower interaction. In: Proceedings of the $6^{\text {th }}$ Symposium and Technical Exhibition on Electromagnetic Compatibility: Zurich, Switzerland 1985.
[11] Podgorski AS, Landt JA. Three dimensional time domain modeling of lightning. IEEE Trans Power Deliv 1987; 2(3): 931-8.

[12] Baba Y, Ishii M. Numerical electromagnetic field analysis of lightning current in tall structures. IEEE Trans Power Deliv 2001; 16 (2): 324-8.

[13] Kordi B, Moini R, Janischewskyj W, Hussein AM, Shostak WO, Rakov VA. Application of the antenna theory model to a tall tower struck by lightning. J Geophys Res 2003; 108 (D17): 4542.

[14] Petrache E, Rachidi F, Pavanello D, et al. Lightning strikes to elevated structures: Influence of grounding conditions on currents and electromagnetic fields. In: Proceedings of IEEE International Symposium on Electromagnetic Compatibility: Chicago, USA 2005.

[15] Rakov VA, Uman MA. Lightning physics and effects: Cambridge University Press 2003.

[16] Cooray V. The lightning flash. IEE Power and Energy Series 34: London, U.K. 2003.

[17] Rakov VA, Uman MA. Review and evaluation of lightning return stroke models including some aspects of their application. IEEE Trans Electromagn Compatibil 1998; 40(4): 403-26.

[18] Kumar U, Raysaha RB, Dileepkumar KP. Time domain modeling of first return stroke of lightning. Open Atmos Sci 2008; 2: 261-70.

[19] Kumar U, Raysaha RB, Dileepkumar KP. Direct time domain modeling of first return stroke of lightning. In: Proceedings of the $29^{\text {th }}$ International Conference on Lightning Protection: Uppsala, Sweden 2008.

[20] Raysaha RB, Kumar U. Review of lightning return stroke models for a direct hit scenario. In: Proceedings of the 2008 Managing Complexity in a Distributed World MCDES: Indian Institute of Science, Bangalore, India 2008.

[21] Little PF. Transmission line representation of a lightning return stroke. J Phys D 1978; 11: 1893-909.

[22] Marcos AM, Christos C. A non-linear transmission line model of the lightning return stroke. IEEE Trans Electromagn Compatibil 1988; 30(3): 401-6.

[23] Nelson T, Cooray V. On the representation of the lightning return stroke process as a current pulse propagating along a transmission line. IEEE Trans Power Deliv 2005, 20(2): 823-37.

[24] Visacro S, De Conti A. A distributed-circuit return-stroke model allowing time and height parameter variation to match lightning electromagnetic field waveform signatures. Geophys Res Lett 2005; 32(23): L23805, 1-L23805, 5.

[25] Podgorski AS. Three dimensional time domain model of lightning including corona effects. In: Proceedings of the International Aerospace and Ground Conference on Lightning and Static Electricity: NASA, Cocoa Beach, Florida, USA 1991.

[26] Moini R, Rakov VA, Uman MA, Kordi B. An antenna theory model for the lightning return stroke. In: Proceedings of the $12^{\text {th }}$ International Zurich Symposium and Technical Exhibition on Electromagnetic Compatibility: Swiss Federal Institute of Technology, Zurich, Switzerland 1997.

[27] Pokharel RK, Ishii M, Baba Y. Numerical electromagnetic field analysis of transient induced voltages associated with lightning to a tall structure. IEEE Trans Electromagn Compatibil 2004; 60(2): 141-7.

[28] Moosavi S, Hassan S, Moini R, Sadeghi SHH. Representation of a lightning return stroke channel as a nonlinearly loaded thin-wire antenna. IEEE Trans Electromagn Compatibil 2009; 51(3): 48898.

[29] Raysaha RB, Kumar U, Thottappillil R. A macroscopic model for first return stroke of lightning. IEEE Trans Electromagn Compatibil 2010; doi: 10.1029/TEMC.2010.2090663.

[30] Raysaha RB, Kumar U, Cooray V, Thottappillil R. Special case of lightning strike to tall objects on ground. In: Proceedings of the $30^{\text {th }}$ International Conference on Lightning Protection: Cagliari, Italy 2010.

[31] Balaram R, Raysaha RB, Kumar U, Cooray V. Analysis for the reflected waves transmitted on to the channel by tall grounded objects using a special case. IEEE Trans Electromagn Compatibil, TEMC-282-2010 (submitted).

[32] Miller EK, Poggio AJ, Burke GJ. An integral equation technique for the time-domain analysis of thin wire structures: The numerical method. J Comput Phys 1973; 12: 24-48.

[33] Raysaha RB, Kumar U. Investigation on the sources of reflection at the junction of tall grounded object and lightning channel. IEEE 
Trans Electromagn Compatibil 2011; doi: 10.1109/TEMC.2011. 2107890.

[34] Rizk FAM. A model for switching impulse leader inception and breakdown of long air-gaps. IEEE Trans Power Deliv 1989; 4(1): 596-606.

[35] Berger K. The Earth Flash. In: Golde RH Eds. Lightning physics of lightning. Academic Press, 1977; vol. 1.
[36] Oetzel GN. Computation of the diameter of a lightning return stroke. J Geophys Res 1968; 73(6): 1889-96.

[37] IEEE Working Group report. Estimating lightning performance of transmission lines I1 - updates to analytical models. IEEE Trans Power Deliv 1993; 8(3): 1254-67.

(C) Raysaha and Kumar; Licensee Bentham Open.

This is an open access article licensed under the terms of the Creative Commons Attribution Non-Commercial License (http://creativecommons.org/licenses/by-nc/ $3.0 /$ ) which permits unrestricted, non-commercial use, distribution and reproduction in any medium, provided the work is properly cited. 